\title{
Projective Collineations of Decomposable Spacetimes Generated by the Lie Point Symmetries of Geodesic Equations
}

\author{
Andronikos Paliathanasis 1,2 (D) \\ 1 Institute of Systems Science, Durban University of Technology, P.O. Box 1334, Durban 4000, South Africa; \\ anpaliat@phys.uoa.gr \\ 2 Instituto de Ciencias Físicas y Matemáticas, Universidad Austral de Chile, Valdivia 5090000, Chile
}

Citation: Paliathanasis, A. Projective Collineations of Decomposable Spacetimes Generated by the Lie Point Symmetries of Geodesic Equations. Symmetry 2021, 13, 1018. https://doi.org/10.3390/sym13061018

Academic Editors: Ugur Camci, Vasilis K. Oikonomou, Bobomurat Ahmedov and Ashfaque H. Bokhari

Received: 15 May 2021

Accepted: 4 June 2021

Published: 6 June 2021

Publisher's Note: MDPI stays neutral with regard to jurisdictional claims in published maps and institutional affiliations.

Copyright: (c) 2021 by the authors. Licensee MDPI, Basel, Switzerland. This article is an open access article distributed under the terms and conditions of the Creative Commons Attribution (CC BY) license (https:/ / creativecommons.org/licenses/by/ $4.0 /)$.

\begin{abstract}
We investigate the relation of the Lie point symmetries for the geodesic equations with the collineations of decomposable spacetimes. We review previous results in the literature on the Lie point symmetries of the geodesic equations and we follow a previous proposed geometric construction approach for the symmetries of differential equations. In this study, we prove that the projective collineations of a $(n+1)$-dimensional decomposable Riemannian space are the Lie point symmetries for geodesic equations of the $n$-dimensional subspace. We demonstrate the application of our results with the presentation of applications.
\end{abstract}

Keywords: projective collineations; Lie symmetries; geodesic equations; decomposable geometries

\section{Introduction}

The theory of Lie point symmetries for the study of differential equations provides a systematic approach for the investigation of similarity solutions for nonlinear dynamical systems. This powerful method established by Sophus Lie at the end of the 19th century [1-3]. The novelty of the S. Lie approach is that he moved the resolution of differential equations from a study of continuous transformations within the context of geometry. The geometric background brought with it the constriction of mapping from a point to a point through transformations. The symmetry analysis has been widely studied in the literature. The simplicity on the steps of the theory and the unexpectedly number of new results which were found the last decades on nonlinear systems, in all areas of applied mathematics [4-17], established the Lie symmetry analysis as one of the most important methods for the study of nonlinear differential equations. Indeed, there are many important results in real world problems which followed by Lie symmetry analysis. An interesting discussion on the Lie symmetries in epidemiology is presented in [18], while the analysis of the differential equations of financial mathematics with the use of Lie symmetries provided a new point of view for this area $[19,20]$. Moreover, an application of Lie's theory in the dynamics of meteorology is discussed in [21].

One of the most important direct result of the Lie point symmetry analysis is the linearization of second-order ordinary differential equations. Sophus Lie on his work proved that for every second-order ordinary differential equation which admits eight Lie point symmetries there exists a transformation in which the given differential equation can be written in the equivalent form of the free particle [1]. Other linearization criteria established in the literature for higher-order differential equations and for partial differential equations [22-25]. In addition, it was found that differential which admit the same symmetries has similar properties, Ovsiannikov carried [26] the first group classification problem and demonstrate the construction of the one-dimensional optimal system for the Lie algebra.

The concept of symmetry is essential in essential in geometry and on physical theories such is general relativity and cosmology. The determination of symmetries in Riemannian manifolds is essential for the derivation of new exact solutions of Einstein's field equations [27-32]. Moreover, the admitted conformal algebra of a manifold is used to perform 
a classification of Riemannian spaces [33,34]. A special class of conformal symmetries are the isometries, which has been used in numerous applications, such as to simplify the gravitational field equations not only in General Relativity $[35,36]$ but in other theories such is the Einstein-Skyrme model [37-39] or to study the geometric properties of the background space, such is the decomposition property of the metric tensor [40].

In Riemannian spaces, the Levi-Civita connection is inherently a system of differential equations defined by the paths of the connection. In terms of differential geometry, these curves remain invariant under the projective collineations. Consequently, there should be a connection between the Lie point symmetries of differential equations with the space collineations. This was the subject of study from different scientific groups, such is that of Katzin et al. [41-43], Aminova [44] and others [45-49]. In [50], Tsamparlis and Paliathanasis found a systematic method for the construction of the Lie point symmetries for the geodesic equations of Riemannian spacetimes by using the elements of the projective algebra for the background space. Furthermore, a similar geometric construction approach was applied for the construction of Noether symmetries [51]. The geodesic Lagrangian Noether symmetries were found to be constructed by the elements of the homothetic algebra for the metric tensor. Some recent extension of this approach on holonomic and unholonomic systems can be found in [52-54].

In this piece of work, previous results on the relation between Lie point and Noether point symmetries for the geodesic equations with the collineations of the background space are reviewed. In Section 2, we present the basic properties and definitions for point transformations and invariant functions. These definitions are extended in the case of geometric objects in Section 3. Moreover, the concept of geometric collineations is discussed, where we focus on the collineations of the metric tensor and of the connection. The Lie point symmetries for differential equations are discussed in Section 4 . In addition, Noether's two theorems are presented. In Section 5 we present two Theorems which gives the explicitly relation of the point symmetries for the geodesic equations with the collineations. Furthermore, in Section 6 we present the new results of this study where we show that the Lie point symmetries for the geodesic equations of $n$-dimensional Riemannian manifold form the projective algebra for a $(n+1)$-dimensional decomposable Riemannian space. Indeed, we prove by using the symmetries of differential equations that the projective algebra of the $(n+1)$-dimensional decomposable space can be constructed by the projective collineations of the $n$-dimensional subspace. Until now, collineations have been used mainly for the construction of the symmetries of differential equations. However, in this study we focus on the inverse approach. Finally in Section 7, we summarize our results and we draw our conclusions.

\section{Point Transformations and Invariant Functions}

Consider $M$ to be a manifold of class $C^{p}$ with $p \succeq 2$ and $U$ to be a neighborhood in $M$. For the two points $P, Q \in U$ with coordinates $\left(x_{P}, y_{P}\right)$ and $\left(x_{Q}^{\prime}, y_{Q}^{\prime}\right)$ respectively, the point transformation on $U$ which drags point $P$ on $Q$ is defined as

$$
x_{Q}^{\prime}=x^{\prime}\left(x_{P}, y_{P}\right), y_{Q}^{\prime}=y^{\prime}\left(x_{P}, y_{P}\right)
$$

in which functions $x^{\prime}(x, y), y^{\prime}(x, y)$ are $C^{p-1}$ and $\operatorname{det}\left|\frac{\partial\left(x^{\prime}, y^{\prime}\right)}{\partial(x, y)}\right| \neq 0$. The later condition is necessary in order the functions $x^{\prime}(x, y), y^{\prime}(x, y)$ to be independent, otherwise the point transformation degenerates. When the point transformation depends on one parameter $\varepsilon$, is called one parameter point transformation [55].

Therefore, if $\Phi$ is the one parameter point transformation $\Phi: P \rightarrow Q$, then

$$
x_{Q}^{\prime}=x^{\prime}\left(x_{P}, y_{P} ; \varepsilon\right), y_{Q}^{\prime}=y^{\prime}\left(x_{P}, y_{P} ; \varepsilon\right) .
$$

By definition, $\Phi$ it admits the group properties, that is, which means that for every function $\Phi$ ant on $P \in U, \Phi: P \rightarrow P^{\prime}$ the resulting point $P^{\prime} \in U$. 
Moreover, the identity transformation exists, $\Phi_{I}: P \rightarrow P$, there exists the associativity, and the inverse element. A group of one parameter point transformation defines a family of curves in $M$, which are parametrized by the parameter $\varepsilon$ and are called the orbits of the group of transformations.

When parameter $\varepsilon$ is infinitesimal we can define the tangent vector a the point $P$ as

$$
X_{P}=\left.\left.\frac{\partial x^{\prime}}{\partial \varepsilon}\right|_{\varepsilon \rightarrow 0} \partial_{x}\right|_{P}+\left.\left.\frac{\partial y^{\prime}}{\partial \varepsilon}\right|_{\varepsilon \rightarrow 0} \partial_{y}\right|_{P}
$$

where now for $\varepsilon^{2} \rightarrow 0$ it follows

$$
x^{\prime}=x+\varepsilon \xi_{P}, \quad y^{\prime}=y+\varepsilon \eta_{P}
$$

where $\xi_{P}=\left.\frac{\partial x^{\prime}}{\partial \varepsilon}\right|_{\varepsilon \rightarrow 0}, \eta=\left.\frac{\partial y^{\prime}}{\partial \varepsilon}\right|_{\varepsilon \rightarrow 0}$.

The vector field $X_{P}$ is called the generator of the infinitesimal point transformation (4). The infinitesimal transformation is the local transformation for the one parameter point transformation (2). The novelty of the point transformations is that for a given local infinitesimal point transformation there can be always defined the global point transformations and vice verse. Indeed, the corresponding point transformation of an infinitesimal transformation can be found by the derivation of integral curve for the infinitesimal generator $X_{P}$.

Consider the function $F(x, y)$ in manifold $M$. Hence, under the action of point transformation $\Phi$ it follows $\Phi: F(x, y) \rightarrow F^{\prime}\left(x^{\prime}(x, y ; \varepsilon), y^{\prime}(x, y ; \varepsilon)\right)$. Hence, function $F$ is invariant under the point transformation $\Phi$ if and only if $F(x, y)=F^{\prime}\left(x^{\prime}(x, y ; \varepsilon), y^{\prime}(x, y ; \varepsilon)\right)$ or $F(x, y)=\lambda\left(F^{\prime}\left(x^{\prime}(x, y ; \varepsilon), y^{\prime}(x, y ; \varepsilon)\right)\right)$ with $F(x, y)=0$, where $\lambda$ is a function, at all points where the one parameter point transformation acts

Equivalently, function, or equation, $F(x, y)=0$, is invariant under the action of an infinitesimal point transformation if the following condition is true

$$
X(F)=0
$$

that is

$$
\xi \frac{\partial F}{\partial x}+\eta \frac{\partial F}{\partial y}=0
$$

The invariant functions of the vector field $X$ are derived from the Lagrange $\frac{d x}{\xi(x, y)}=$ $\frac{d y}{\eta(x, y)}$. Hence, every function of the form $F(x, y)=F(W)$, in which $W$ is the zero-order invariant $d W=\frac{d x}{\xi(x, y)}-\frac{d y}{\eta(x, y)}$, is invariant under the infinitesimal transformation with generator the vector field $X$.

Furthermore, the vector field $X$ is called Lie point symmetry for the function $F(x, y)$. Since, the one parameter point transformations form a group, then the infinitesimal generators $\left\{X_{1}, X_{2}, \ldots, X_{N}\right\}$ which are symmetry vectors for function $F(x, y)$ form a Lie algebra.

Until this point we have discussed the Lie point symmetries for functions. In the following, we focus with the application of point transformations on differential equations and on geometric objects in Riemannian manifolds.

\section{Geometric Objects and Collineations}

Let $\Omega$ be a geometric object of class $r$ on the $C^{p}$ manifold $M$, with $r \leq p$. Then $\Omega$ is well defined on a every point $P$, that is $\Omega^{a}=\Omega^{a}(x, y)$. Additionally, under a coordinate transformation $(x, y)=J^{i}(x, y)$ the new components $\Omega^{a^{\prime}}$ of the object in the new coordinates $\left\{x^{\prime} y^{\prime}\right\}$ are represented as well determined functions of class $r^{\prime}=p-r$ of the old components $\Omega^{a}$ in the old coordinates $\{x, y\}$, of the functions $J^{i}$ and of their s-th derivatives $(1 \leq s \leq p)$, that is, the new components $\Omega^{i^{\prime}}$ of the object can be represented by equations of the form $\Omega^{a^{\prime}}=\Phi^{a}\left(\Omega^{k}, x, y, x^{\prime}, y^{\prime}\right)$, where functions $\Phi^{a}$ have the group 
properties. The transformation law, i.e. functions $\Phi^{a}$ characterize the geometric object. In the following we are interesting on differential geometric objects in which

$$
\Phi^{a}\left(\Omega, x^{k}, x^{k^{\prime}}\right)=J_{b}^{a}\left(x, y, x^{\prime}, y^{\prime}\right) \Omega^{b}+C\left(x, y, x^{\prime}, y^{\prime}\right) .
$$

and on the special case with $C\left(x^{k}, x^{k^{\prime}}\right)$, where $\Omega^{a}$ are known as tensors and $J_{b}^{a}\left(x, y, x^{\prime}, y^{\prime}\right)$ is the Jacobian tensor.

Consider now the infinitesimal transformation (4) with generator the vector field $X$. Then, under the map $\Phi$ the geometric object differs as

$$
\mathcal{L}_{X} \Omega=\lim _{\varepsilon \rightarrow 0} \frac{1}{\varepsilon}[\Phi(\Omega)-\Omega] .
$$

Operator $\mathcal{L}_{X} \Omega$ is the Lie derivative with respect to the vector field $X$ on the geometric object $\Omega$. In terms of coordinates the definition of Lie derivative depends on the transformation law $\Phi^{a}$.

Indeed, for a function $F(x, y)$, the Lie derivative is defined as $\mathcal{L}_{X} F=X(F)$. Furthermore, for tensor field $T$ of rank $(r, s)$ is defined as follows [56]

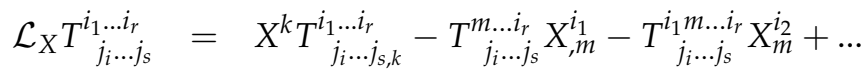

$$
\begin{aligned}
& \ldots+T_{{ }_{m \ldots} \ldots j_{s}}^{i_{1} \ldots i_{r}} X_{, j_{1}}^{m}+T_{j_{i} m \ldots j_{s}}^{i_{1} \ldots i_{r}} X_{j_{2}}^{m}+\ldots
\end{aligned}
$$

where the Einstein summation convention is considered.

On the other hand, the connection coefficients have different transformation law from tensor fields, that means that the Lie derivative is defined different. Specifically, the definition of $\mathcal{L}_{X} \Gamma_{j k}^{i}$ is

$$
\mathcal{L}_{X} \Gamma_{j k}^{i}=X_{, j k}^{i}+\Gamma_{j k, r}^{i} X^{r}-X_{, r}^{i} \Gamma_{j k}^{r}+X_{, j}^{s} \Gamma_{s k}^{i}+X_{, k}^{s} \Gamma_{j s}^{i} .
$$

When we have a symmetric connection $\Gamma_{j k}^{i}=\Gamma_{k j}^{i}$ the latter expression can be written in the equivalent form

$$
\mathcal{L}_{X} \Gamma_{j k}^{i}=X_{; j k}^{i}-R_{j k l}^{i} \xi^{l},
$$

in which $R_{j k l}^{i}$ is the curvature tensor and the semicolon ";" means covariant derivative.

Previously, the invariant functions under point transformations were defined. In the folloiwng lines, we can easily extend the same definition in the case of geometric objects. Therefore, it will be said that a geometric object $\Omega$ is invariant under the action of the one parameter point transformation (2) , $\Phi: \Omega \rightarrow \Omega^{\prime}$, if and only if $\Omega^{\prime}\left(x^{\prime}, y^{\prime}\right)=\Omega(x, y)$. The later conditions are expressed in terms of the infinitesimal transformation (4) as $L_{\tilde{\zeta}} \Omega=0$.

However, in terms of geometric objects the concept of symmetry has been generalized, such that a geometric $\Omega$ object under the action of a point transformation to differs by a tensor $\Psi$, that is, the generalized conditions $\mathcal{L}_{X} \Omega=\Psi$. While $\Omega$ can be any geometric object with arbitrary transformation law, the Lie derivative $\mathcal{L}_{X} \Omega$ has the same transformation law with a tensor which means that $\Psi$ is always a tensor field. Vector fields $X$ which satisfy such relations are called geometric collineations. In the case of Riemannian manifolds, the most common collineations are these of the metric tensor and of the connection coefficients.

\subsection{Collineations of the Metric Tensor}

Assume a Riemannian space $V^{n}$ of dimension $n, \operatorname{dim} V^{n}=0$, with metric tensor $g_{i j}$ and connection coefficient $\Gamma_{j k}^{i}$. Then a vector field $X$ is characterized as a Conformal Killing vector (CKV) for the Riemannian space if

$$
\mathcal{L}_{X} g_{i j}=2 \psi g_{i j}, \psi=\frac{1}{n} X_{; k}^{k} .
$$


CKVs have the property to keep invariant the angle between two directions at a point, under the action of the point transformations. The CKVs of a given metric tensor $g_{i j}$ form a Lie algebra known as conformal algebra for the space $V^{n}$. There are some important properties for the conformal algebra. For instance, conformal related metrics, i.e., $\bar{g}_{i j}, g_{i j}$ with $\bar{g}_{i j}=N g_{i j}$ admit the same conformal algebra, while the maximum dimension of the admitted conformal algebra can be $\frac{1}{2}(n+1)(n+2)$. In that case the space with line element $g_{i j}$ is characterized as conformally flat and there exists a coordinate system where it can be written as $g_{i j}=N \eta_{i j}$, where $\eta_{i j}$ notes the metric tensor for the flat space.

In the special case where function $\psi$ is constant, that is, $\psi_{, i}=0$, the CKV is reduced to the Homothetic Killing vector (HKV). A Riemannian space can admits at maximum one proper HKV. Finally, when $\psi=0$, the vector field $X$ is called a Killing vector field or isometry.

Isometries are the most important symmetries in geometries because they keep invariant the distances and the angles in a Riemannian space. The most important isometries are the translations and the rotations of Euclidean space. Because of these symmetries the geometric objects do not change when they rotate or change location in the physical space.

The KVs form a Lie algebra known as Killing algebra of maximum dimension $\frac{1}{2} n(n+1)$. In the later case, the space $g_{i j}$ is maximally symmetric and it is that of the flat space or of the $n$-dimensional sphere or of the $n$-dimensional hyperbolic plane. If the maximally symmetric space admits a (proper) HKV then it is a flat space. Maximally symmetric spaces are conformally flat which means that they admit the maximum conformal Lie algebra of dimension $\frac{1}{2}(n+1)(n+2)$.

\subsection{Collineations of the Connection}

Let us assume the Levi-Civita connection $\Gamma_{j k}^{i}$, then by definition, the Lie derivative with respect to the field $X$ is written as

$$
\mathcal{L}_{X} \Gamma_{j k}^{i}=g^{i r}\left[\left(\mathcal{L}_{X} g_{r k}\right)_{; j}+\left(\mathcal{L}_{X} g_{r j}\right)_{; k}-\left(\mathcal{L}_{X} g_{j k}\right)_{; r}\right] .
$$

Hence, if $X$ is a CKV from (13) it follows

$$
\mathcal{L}_{X} \Gamma_{j k}^{i}=2 g^{i r}\left[\psi_{; j}+\psi_{; k}-\psi_{; r}\right]
$$

Therefore, if $X$ is a HKV or a KV, it follows $\mathcal{L}_{X} \Gamma_{j k}^{i}=0$, which means that the LeviCivita connection remain invariant under the action of Killing symmetry and of Homothetic symmetry. However, that is not true for a proper Conformal symmetry. The collineations of the connection can be defined independently from the collineations of the metric tensor. (AC) if

Consider now the general connection $\Gamma_{j k}^{i}$, then the vector field $X$ is an Affine collineation

$$
L_{\xi} \Gamma_{j k}^{i}=0,
$$

This family of point transformations caries geodesic trajectories into geodesic trajectories while preserve the affine parameter of the geodesic equations. ACs form the so-called Affine Lie algebra and in the case of the Levi-Civita connection the Affine Lie algebra has the subalgebras the Homothetic algebra and the Killing algebra. The maximum dimension of the Affine algebra is $n(n+1)$ which is that of the flat space.

Another family of collineations for the connection of special interest are the Projective collineations (PC). Projective transformations transform the system of geodesics (auto parallel curves) of $V^{n}$ into the same system but they do not preserve the affine parameter. A vector field $X$ is called a PC if there exists an one-form $\omega_{i}$ where

$$
L_{\xi} \Gamma_{j k}^{i}=\omega_{j} \delta_{k}^{i}+\omega_{k} \delta_{j}^{i}
$$


However, in the case of Riemannian manifolds the one-form $\omega_{i}$ is always closed, i.e., $\omega_{i}=\phi_{, i}$ which means that the later symmetry condition is equivalent to

$$
L_{\zeta} \Gamma_{j k}^{i}=\phi_{, j} \delta_{k}^{i}+\phi_{, k} \delta_{j}^{i}
$$

Function $\phi$ is called the projective function. When $\phi$ vanishes the PC reduces to that of AC while when $\phi_{; i j}=0$ the PC is characterized as special. The maximum dimension of the projective algebra is $n(n+2)$, and it is that of the maximal symmetric spaces [57].

As far as the existence of a special PC is concerned, there are some important results in the literature which are necessary for our study [57]. Indeed, if the Riemannian space admits a $p \leq n$ dimensional Lie algebra of special PCs then also admits $p$ gradient KVs and a gradient HV and if $p=n$ the space is flat, the reverse also holds true. Furthermore, a maximally symmetric space which admits a proper AC or a special PC is a flat space.

There is a zoology of definitions for collineations in Riemannian spaces, we refer the reader in the interesting diagram in [58].

At this point it is important to mention that with the term gradient collineation we refer to a collineation vector field $X$, which can be written as a closed one-form. Moreover, with the term proper collineation we refer to a collineation with a specific property. For instance as a proper $\mathrm{AC}$ we refer to an $\mathrm{AC}$ which is not a $\mathrm{KV}$ or a $\mathrm{HKV}$, while with the term proper $\mathrm{PC}$ we refer to a collineation which is a PC and not a AC or HKV or KV.

\section{Symmetries of Ordinary Differential Equations}

Below the application of point symmetries on differential equations is presented. We give the definition of a Lie point symmetry for differential equations and we show how the Lie invariants are applied to simplify a given differential equation.

A differential equation $H=0$ is a function defined in the jet space $B_{M}=\left\{x, y, \dot{y}, \ddot{y} \ldots, y^{(n)}\right\}$, where we have assumed $x$ to be the independent variable and $y=y(x)$ to be the dependent variable and $\dot{y}=\frac{d y}{d x}, \ddot{y}=\frac{d^{2} y}{d x^{2}}, \ldots, y^{(n)}=\frac{d^{(n)} y}{d x^{n}}$. Therefore function $H$ is defined as $H=H\left(x, y, \dot{y}, \ddot{y} \ldots, y^{(n)}\right)$.

Consider now the infinitesimal transformation in the basic manifold $\{\bar{x}, \bar{y}\} \rightarrow\{x+\varepsilon \xi(x, y), y+\varepsilon \eta(x, y)\}$, where in the jet space $B_{M}$ the point transformation is prolonged as

$$
\begin{aligned}
\bar{x}= & x+\varepsilon \xi(x, y) \\
\bar{y}= & x+\varepsilon \eta(x, y) \\
\bar{y}^{(1)}= & y^{(1)}+\varepsilon \eta^{[1]} \\
& \cdots \\
\bar{y}^{(n)}= & y^{(n)}+\varepsilon \eta^{[n]}
\end{aligned}
$$

with generator vector

$$
X^{[n]}=\xi \partial_{x}+\eta \partial_{\eta}+\eta^{[1]} \partial_{\dot{y}}+\ldots+\eta^{[n]} \partial_{y^{(n)}} .
$$

Functions $\eta^{[n]}$ are known as the prolongation/extensions functions and they are defined as

$$
\begin{aligned}
\eta^{[1]}= & \dot{\eta}-y^{(1)} \dot{\xi}, \\
\eta^{[2]}= & \dot{\eta}^{[1]}-\ddot{y} \dot{\xi}, \\
& \cdots \\
\eta^{[n]}= & \frac{d \eta^{n-1}}{d x}-y^{(n)} \frac{d \xi}{d x}=\frac{d^{n}}{d x^{n}}\left(\eta-y^{(1)} \xi\right)+y^{(n+1)} \xi .
\end{aligned}
$$


Therefore, the differential equation $H\left(x, y, \dot{y}, \ddot{y} \ldots, y^{(n)}\right)=0$ is invariant under the action of an parameter point transformation if the following condition is true

$$
X^{[n]}\left(H\left(x, y, \dot{y}, \ddot{y} \ldots, y^{(n)}\right)\right)=0,
$$

or equivalently

$$
X^{[n]}\left(H\left(x, y, \dot{y}, \ddot{y} \ldots, y^{(n)}\right)\right)=\lambda\left(H\left(x, y, \dot{y}, \ddot{y} \ldots, y^{(n)}\right)\right) \text {, with } H=0 .
$$

Hence, when the symmetry condition (22) is true, the infinitesimal generator $X$ will be called Lie point symmetry for the differential equation. The basic application of the Lie point symmetries are summarized in the application of the similarity transformations which are used to reduce the order on differential equations or the number of dependent variables for partial differential equations.

There are different ways to apply the Lie point symmetries for the reduction of a given differential equation. However, the different approaches are equivalent. The most common methods are the derivation of canonical transformations, and the derivation of the Lie invariants.

Let $X=\xi(x, y) \partial_{x}+\eta(x, y) \partial_{y}$ be a Lie point symmetry for the differential equation $H\left(x, y, \dot{y}, \ddot{y}_{\ldots,} y^{(n)}\right) \equiv y^{(n)}-\omega\left(x, y, \dot{y}, \ldots, y^{(n-1)}\right)=0$. Then under the change of variables $\{x, y\} \rightarrow\{r, s\}$ such that

$$
X r=0, X s=1
$$

the symmetry vector reads $X=\partial_{s}$, while the differential equation as

$$
\frac{d^{n} s}{d r^{2}}=\bar{\omega}\left(r, s, \frac{d s}{d r}, \ldots, \frac{d^{n-1} s}{d r^{n-1}}\right)
$$

By definition it follows $\frac{\partial}{\partial s} \bar{\omega}\left(r, s, \frac{d s}{d r}, \ldots, \frac{d^{n-1} s}{d r^{n-1}}\right)=0$, therefore by define the new variable $S=\frac{d s}{d r}$, we are able to reduce the order of the differential equation and rewrite it as

$$
\frac{d^{n-1} S}{d r^{n-1}}=\bar{\omega}\left(r, S, \frac{d S}{d r}, \ldots, \frac{d^{n-2} S}{d r}\right) .
$$

The second approach that we discuss for the application of the Lie point symmetries for the reduction of a differential equation is based on the derivation of the differential invariant functions which follows from the Lagrange system

$$
\frac{d x}{\xi}=\frac{d y}{\eta}=\frac{d \dot{y}}{\eta_{[1]}}=\ldots=\frac{d y^{(n)}}{\eta_{[n]}} .
$$

The system (27) provides us with characteristic functions

$$
W^{[0]}(x, y), W^{[1]}(x, y, \dot{y}), W^{[n]}\left(x, y, \dot{y}, \ddot{y}, \ldots, y^{(n)}\right)
$$

where $W^{[n]}$ is the nth order invariant of the Lie symmetry vector. By considering as $u=W^{[0]}$ to be the new independent variable and $v=W^{[1]}$ the new dependent variable then the differential equation $H\left(x, y, \dot{y}, \ddot{y} \ldots, y^{(n)}\right) \equiv y^{(n)}-\omega\left(x, y, \dot{y}, \ldots, y^{(n-1)}\right)=0$ can be written in the equivalent form

Let $u=W^{[0]}, v=W^{[1]}$, where $W^{[0]}, W^{[1]}$ are the zero and the first order invariants of a Lie symmetry repetitively. From zero-order and first-order invariants $u, v$, the higherorder differential invariants

$$
\frac{d v}{d u}, \ldots, \frac{d^{n-1} v}{d u^{n-1}}
$$

can be defined. 
The differential invariants are functions of the derivatives $y^{(n)}$. Hence, the given differential given $H\left(x, y, \dot{y}, \ddot{y} \ldots, y^{(n)}\right)=0$, may be written in terms of the differential invariants (28), i.e.,

$$
\frac{d^{n-1} v}{d u^{n-1}}=\Omega\left(u, v, \frac{d v}{d u}, \ldots \frac{d^{n-1} v}{d u^{n-1}}\right)
$$

where $\frac{d v}{d u}=\frac{\frac{\partial v}{\partial x}+\frac{\partial v}{\partial y} \dot{y}+\ldots+\frac{\partial v}{\partial y} \ddot{y}}{\frac{\partial v}{\partial x}+\frac{\partial v}{\partial y} \dot{y}}$.

There are alternative methods and approaches to apply Lie symmetries. A quite intriguing application of Lie symmetries is to produce integrals or Lagrangian functions for a system of ODEs by the method of Jacobi's last multiplier, see for instance [59], while there are very interesting approaches for the construction of conservation laws by using the Lie point symmetries $[60,61]$.

The most famous approach for the derivation of conservation laws from the point symmetries for systems with an Action principle is described by Noether's theorems. Noether's theorem for second-order differential equations $H(x, y, \dot{y}, \ddot{y})=0$ is presented.

Let function $L=L(x, y, \dot{y})$ be the Lagrangian for the differential equation $H(x, y, \dot{y}, \ddot{y})=0$, that is, $E_{L}(L)=H(x, y, \dot{y}, \ddot{y})$, where $E_{L}$ is the Euler-Lagrange vector. Therefore, if the following condition is true

$$
X^{[1]} L+L \frac{d \xi}{d x}=\frac{d f}{d x}
$$

where $f$ is a function, then the variation of the Action integral is invariant. The later it means that $X$ is a Lie point symmetry for the differential equation $H(x, y, \dot{y}, \ddot{y})=0$, while $X$ is called a Noether symmetry for the Lagrangian function $L(x, y, \dot{y})$.

Moreover, from Noether's second theorem it follows that for every Noether symmetries $X$ the following function is a conservation laws for the differential equation

$$
\Phi(x, y, \dot{y})=\xi(x, y)\left(\dot{y} \frac{\partial L}{\partial \dot{y}}-L\right)-\eta(x, y) \frac{\partial L}{\partial y}+f,
$$

that is, $\frac{d \Phi}{d x}=0$.

Noether symmetries for a given dynamical system form a Lie algebra known as Noether algebra. The Noether algebra is a subalgebra of the admitted Lie algebra for the dynamical system.

\section{Symmetries of Geodesic Equations}

We continue our analysis by studying the Lie point symmetries for the geodesic equations. In particular, we review previous results which connect the Lie point symmetries of geodesic trajectories with the collineations of the background space. Because the following applications are of special interests in physics $t$ is assumed to be the independent variable and $x^{i}=x^{i}(t)$ to be the dependent variables.

Let as assume the $C^{p}$ manifold of dimension $n$ with the connection $\Gamma_{j k}^{i}$. Thus, in a local coordinate system the autoparallels are defined as

$$
\ddot{x}^{i}+\Gamma_{j k}^{i} \dot{x}^{j} \dot{x}^{k}+\phi(t) \dot{x}^{i}=0
$$

where $t$ is a parameter along the paths, and a dot means derivative with respect to $\mathrm{s}$. When $\phi(t)$ vanishes, the autoparallels are affine parametrized and $t$ is the affine parameter.

Without loss of generality a new variable $d t=f(t) d t$ can always be defined such that Equation (32) to be written as

$$
\frac{d^{2} x^{i}}{d S^{2}}+\Gamma_{j k}^{i} \frac{d x^{j}}{d S} \frac{d x^{k}}{d S}+\left(\frac{d S}{d t}\right)^{-2}\left(\left(\frac{d^{2} S}{d t^{2}}\right)+\phi(S)\left(\frac{d S}{d S}\right)\right) \frac{d x^{i}}{d S}=0
$$


or

$$
\frac{d^{2} x^{i}}{d S^{2}}+\Gamma_{j k}^{i} \frac{d x^{j}}{d S} \frac{d x^{k}}{d S}=0
$$

where we have set $\frac{d f(t)}{d t}+\phi(t) f(t)=0$. Therefore in the following it is assumed $\phi(t)=0$.

Consider the infinitesimal transformation

$$
\bar{t}=s+\varepsilon \xi\left(s, x^{k}\right), \quad \bar{x}^{i}=x^{i}+\varepsilon \eta^{i}\left(t, x^{k}\right)
$$

with generator $X=\xi\left(t, x^{k}\right) \partial_{t}+\eta^{i}\left(t, x^{k}\right) \partial_{i}$.

The autoparallels (32) are invariant under the transformation (35) iff the following conditions holds

$$
X^{[2]}\left(\ddot{x}^{i}+\Gamma_{j k}^{i} \dot{x}^{j} \dot{x}^{k}\right)=0
$$

by replacing $X^{[2]}$ from its definition and consider the coefficients of polynomial of the derivatives of $x^{i}$ to be equal with zero follows the following system

$$
\begin{gathered}
\eta_{, t t}^{i}=0 \\
\xi_{, t t} \delta_{j}^{i}-2\left[\eta_{, t j}^{i}+\eta_{, t}^{k} \Gamma_{(k j)}^{i}\right]=0 \\
\mathcal{L}_{\mathbf{j}} \Gamma_{(j k)}^{i}=\xi^{\Gamma^{i}}{ }_{(k j), t}^{i}+2 \xi_{, t(j)} \delta_{k)}^{i} \\
\xi_{(, j \mid k} \delta_{d)}^{i}=0 .
\end{gathered}
$$

We proceed with the solution of the symmetry conditions (37)-(40). In addition we consider that the connection coefficients they do not depend on the indepedent parameter $t$.

From Equations (37) and (40) we derive $\eta^{i}\left(t, x^{k}\right)=A^{i}\left(x^{k}\right) t+B^{i}\left(x^{k}\right)$ and $\xi(t, x)=$ $C_{J}(t) S^{J}\left(x^{k}\right)+D(t)$. Functions $A^{i}\left(x^{k}\right), B^{i}\left(x^{k}\right), C_{J}(t), D(t)$ are arbitrary functions which will be constraint by the symmetry conditions (38), (39) while $S^{J}\left(x^{k}\right)$ is the generating function for a gradient KV, i.e., $S^{J}(x)_{\mid(i, j)}=0$. Index $J$ run on the number of independent gradient KVs.

Furthermore, by replacing in (38) it follows

$$
2 A\left(x^{k}\right)_{; j}^{i}=\left[C_{J}(t), t t S^{J}\left(x^{k}\right)+D(t), t t\right] \delta_{j}^{i},
$$

that is;

$$
\begin{aligned}
D(t) & =\frac{1}{2} M t^{2}+K t+L \\
C_{J}(t) & =\frac{1}{2} G_{J} t^{2}+E_{J} t+F_{J}
\end{aligned}
$$

in which $M, K, L, G_{J}, E_{J}, F_{J}$ are constants.

In addition, for the function $A^{i}\left(x^{k}\right)$, from (41) we derive $A\left(x^{k}\right)_{i ; j}=\frac{1}{2}\left(G_{J} S^{J}\left(x^{k}\right)+M\right) g_{i j}$, where $g_{i j}$ is the background metric tensor. Consequently, $A^{i}\left(x^{k}\right)$ is an element of the conformal algebra for the metric tensor $g_{i j}$. However, antisymmetric part vanishes, i.e., $A\left(x^{k}\right)_{[i ; j]}=0$, which means that $A^{i}\left(x^{k}\right)$ is a gradient CKV with conformal factor $\psi=\frac{1}{2}\left(G_{J} S^{J}(x)+M\right)$.

Finally, from the symmetry condition (39) we end with the following system

$$
\begin{aligned}
L_{A} \Gamma_{j k}^{i} & =2 G_{J} S^{J}(x)_{,(j} \delta_{k)}^{i}, \\
L_{B} \Gamma_{j k}^{i} & =2 E_{J} S^{J}(x)_{,(j} \delta_{k)}^{i},
\end{aligned}
$$


that is, the vector fields $A^{i}\left(x^{k}\right), B^{i}\left(x^{k}\right)$ are special PCs with projective functions $G_{J} S^{J}(x)$ and $E_{J} S^{J}(x)$ respectively. However, because $A^{i}\left(x^{k}\right)$ is also an element of the conformal group for the underlying metric, it follows that $A^{i}\left(x^{k}\right)$ is a gradient KV or a gradient HV.

We conclude that the Lie point symmetries for the geodesic equations, or for the autoparallels, are constructed by the elements of the special projective algebra of the background space as described by the following theorem

Theorem 1. The generic Lie point symmetry vector $X=\xi\left(t, x^{k}\right) \partial_{t}+\eta^{i}\left(t, x^{k}\right) \partial_{i}$ for the geodesic equations

$$
\ddot{x}^{i}+\Gamma_{j k}^{i}\left(x^{k}\right) \dot{x}^{j} \dot{x}^{k}=0
$$

in a Riemannian background space with metric tensor $g_{i j}\left(x^{k}\right)$, where $\Gamma_{j k}^{i}\left(x^{k}\right)$ is the Levi-Civita connection, is generated by the elements of the special projective algebra for the Riemannian manifold.

When the background space admits gradient KVs $S^{J}\left(x^{k}\right)$, functions $\xi\left(t, x^{k}\right), \eta^{i}\left(t, x^{k}\right)$ are given by the formula

$$
\begin{gathered}
\xi\left(t, x^{k}\right)=\frac{1}{2}\left(G_{J} S^{J}\left(x^{k}\right)+M\right) t^{2}+\left[E_{J} S^{J}\left(x^{k}\right)+K\right] t+F_{J} S^{J}\left(x^{k}\right)+L, \\
\eta^{i}\left(t, x^{k}\right)=A^{i}\left(x^{k}\right) t+B^{i}\left(x^{k}\right)+D^{i}\left(x^{k}\right)
\end{gathered}
$$

where $G_{J}, M, b, K, F_{J}$ and $L$ are constants and the index $J$ runs along the number of gradient $K V$, $A^{i}(x)$ is a gradient $H V$ with conformal factor $\psi=\frac{1}{2}\left(G_{J} S^{J}\left(x^{k}\right)+M\right), D^{i}(x)$ is a non-gradient $K V$ of the metric and $B^{i}(x)$ is either a special projective collineation with projection function $E_{J} S^{J}(x)$ or an $A C$ and $E_{J}=0$.

When the background space does not admit gradient $K V s$, functions $\xi\left(t, x^{k}\right), \eta^{i}\left(t, x^{k}\right)$ are given by the formula

$$
\begin{gathered}
\xi\left(t, x^{k}\right)=\frac{1}{2} M t^{2}+K t+L \\
\eta^{i}\left(t, x^{k}\right)=A^{i}\left(x^{k}\right) t+B^{i}\left(x^{k}\right)+D^{i}\left(x^{k}\right),
\end{gathered}
$$

where $A^{i}(x)$ is a gradient $H V$ with conformal factor $\psi=\frac{1}{2} M, D^{i}(x)$ is a non-gradient $K V$ of the metric and $B^{i}(x)$ is an $A C$.

Furthermore, when the metric tensor does not admit gradient $K V$ and gradient $H K V$, functions $\xi\left(t, x^{k}\right), \eta^{i}\left(t, x^{k}\right)$ are given by the formula

$$
\begin{aligned}
\xi(t) & =K t+L \\
\eta^{i}\left(x^{k}\right) & =B^{i}\left(x^{k}\right)+D^{i}\left(x^{k}\right) .
\end{aligned}
$$

Finally, if the background vector field admits a zero-dimensional special projective algebra the generic Lie point symmetry is $X=(K t+L) \partial_{t}$.

We proceed our discussion with the investigation of the Noether symmetries for the geodesic equations. Because Noether symmetries form a subalgebra of the Lie symmetries, Noether vector fields are constructed by the elements of the special projective algebra for the background space.

Consider the geodesic Lagrangian function

$$
L\left(x^{k}, \dot{x}^{k}\right)=\frac{1}{2} g_{i j}\left(x^{k}\right) \dot{x}^{i} \dot{x}^{j} .
$$


Then, from the Noether symmetry condition (30) for the infinitesimal generator $X=\xi\left(t, x^{k}\right) \partial_{t}+\eta^{i}\left(t, x^{k}\right) \partial_{i}$, we end with the following system of partial differential equations

$$
\begin{aligned}
\xi_{, k} & =0 \\
\mathcal{L}_{\eta} g_{i j} & =2\left(\frac{1}{2} \xi_{, t}\right) g_{i j} \\
\eta_{, t}^{, i} g_{i j} & =f_{, i} \\
f_{, t} & =0
\end{aligned}
$$

From (55) it follows $\xi\left(t, x^{k}\right)=\xi(t)$, while condition (54) gives $\eta_{i}(t, x)=f, i\left(t, x^{k}\right) t+$ $K_{i}\left(x^{j}\right)$. Additionally, equation (53) provides that $\xi_{, t}=2 \psi$, where $\psi=$ const. while $\eta^{i}\left(t, x^{k}\right)$ is HKV for the background space. By following the same steps with as in the case of Lie point symmetries, for the Noether point symmetries for the geodesic Lagrangian our results are summarized in the following theorem

Theorem 2. The Noether Symmetries of the geodesic Lagrangian (51) are generated by the KVs and the HKV of the metric $g_{i j}$ as follows:

$$
\begin{aligned}
X & =\left(C_{3} \psi t^{2}+2 C_{2} \psi t+C_{1}\right) \partial_{t}+ \\
& +\left[C_{J} S^{J, i}+C_{I} K V^{I i}+C_{I J} t S^{J, i}+C_{2} H^{i}+C_{3} t(G H V)^{i}\right] \partial_{i}
\end{aligned}
$$

with corresponding gauge function

$$
f\left(x^{k}\right)=C_{1}+C_{2}+C_{I}+C_{J}+\left[C_{I J} S^{J}\right]+C_{3}[G H V],
$$

where $S^{J, i}$ are the $C_{J}$ gradient $K V s, K V^{I i}$ are the $C_{I}$ non-gradient $K V s, H^{i}$ is a $H K V$ not necessarily gradient $[G H V]$ and is the gradient $H K V$ (if it exists) of the metric $g_{i j}$.

Finally, from Noether's second theorem and Theorem 2 for the conservation laws of the geodesic equations it follows the theorem.

Theorem 3. The generic form of the Noetherian conservation law for the geodesic Lagrangian (51) is

$$
\begin{aligned}
\phi & =\frac{1}{2}\left[C_{3} \psi t^{2}+2 C_{2} \psi t+C_{1}\right] g_{i j} \dot{x}^{i} \dot{x}^{j} \\
& +\left[C_{J} S^{J, i}+C_{I} K V^{I i}+C_{I J} t S^{J, i}+C_{2} H^{i}\left(x^{r}\right)+C_{3} t(G H V)^{, i}\right] g_{i j} \dot{x}^{j} \\
& +C_{1}+C_{2}+C_{I}+C_{J}+\left[C_{I J} S^{J}\right]+C_{3}[G H V] .
\end{aligned}
$$

The individual conservation laws linear in the momentum are

$$
\begin{aligned}
C_{I} & \neq 0: \phi_{C_{I}}=K V_{i}^{I} \dot{x}^{i}-1, \\
C_{J} & \neq 0: g_{i j} S^{J, i} \dot{x}^{j}-1, \\
C_{I J} & \neq 0: g_{i j} S^{J, i} \dot{x}^{j}-S^{J},
\end{aligned}
$$


while the quadratic in the momentum conservation laws are

$$
\begin{aligned}
& C_{1} \neq 0: \phi_{C_{1}}=\frac{1}{2} g_{i j} \dot{x}^{i} \dot{x}^{j}+1, \\
& C_{2} \neq 0: \phi_{C_{2}}=t \psi g_{i j} \dot{x}^{i} \dot{x}^{j}-g_{i j} H^{i} \dot{x}^{j}+1, \\
& C_{3} \neq 0: \phi_{C_{3}}=\frac{1}{2} t^{2} \psi g_{i j} \dot{x}^{i} \dot{x}^{j}-t(G H V)_{, i} \dot{x}^{i}+[G H V] .
\end{aligned}
$$

From Theorems 1 and 2 it is clear that one is able to compute the Lie symmetries and the Noether symmetries of the geodesic equations in Riemannian manifolds by derive the collineation vectors and avoid the cumbersome formulation of the Lie symmetry method. However, the inverse procedure is true. In the following, we focus on the inverse approach in order to construct projective algebra of decomposable spacetimes by the Lie point symmetries of the nondecomposable part of the space.

\section{Projective Collineations of Decomposable Spacetimes}

The symmetry condition (39) can be written in the equivalent form

$$
\mathcal{L}_{\eta} \Gamma_{(j k)}^{i}+\mathcal{L}_{\xi} \Gamma_{(j k)}^{i}=2 \Phi_{(, j} \delta_{k)}^{i}
$$

in which $\Phi=\xi, t$. Furthermore, if we sum the symmetry conditions (37)-(40) we end with the equation

$$
\mathcal{L}_{X} \Gamma_{B C}^{A}=2 \Phi_{(, A} \delta_{C)}^{B}
$$

where $\Gamma_{B C}^{A}$ is the Levi-Civita connection for the decomposable Riemannian manifold

$$
d s^{2}=\varepsilon d t^{2}+g_{i j}\left(x^{k}\right) d x^{i} d x^{j},
$$

with nonzero components $\Gamma_{B C}^{A}=\Gamma_{j k}^{i}$ in which $A, B$ are the indices in the $1+n$ spaces and $i, j$ the indices in the $n$-space. Thus, the following theorem for the collineations of decomposable spaces follows.

Theorem 4. The Lie point symmetries for the geodesic equations of the n-dimensional Riemannian space $g_{i j}\left(x^{k}\right)$ form the projective algebra for the $(n+1)$-decomposable Riemannian space $(67)$ and vice versa.

From Theorem 4 and the function forms of the Lie point symmetries as they are given in Theorem 1 we end with the following corollarytheorem.

Theorem 5. For the elements of the special projective algebra of the decomposable space (67) we have the following observations:

(A) The $(n+1)$-dimensional decomposable space admits a proper PC, then the field is a special projective collineation.

(B) The $(n+1)$-dimensional decomposable space admits a HKV if and only if the n-space admits a HKV.

(C) The $(n+1)$-dimensional decomposable space admits a proper PC if and only if the $n$-space admits a gradient $H K V$.

(D) There exists a rotation among the axes $t$ and $x^{i}$ if and only if there exists a gradient $K V$ on the direction of $x^{i}$.

(E) The $(n+1)$-dimensional decomposable space admits a projective algebra $G^{n+1}$ of minimum dimension 2. When $\operatorname{dim} G^{n+1}=2$, then $n$-dimensional space does not admit any special PC.

Some of the above results have been proved before in the literature by using tools of differential geometry. However, in our approach we applied tools from the theory of 
symmetries of differential equations. We demonstrate the application of Theorem 4 with some examples.

Consider the space of constant curvature

$$
d \tau^{2}=\frac{1}{\left(1+\frac{1}{4} K x^{i} x_{i}\right)^{2}}\left(d x^{2}+d y^{2}+d z^{2}\right)
$$

with $K \neq 0$. The space admits a six elements on the special projective algebra consisted by the (nongradient) Killing symmetries $\mathbf{r}_{\mu v}, \mathbf{I}_{\mu}$ [50]. The Lie point symmetries for the geodesic equations of the space are the vector fields

$$
\mathbf{r}_{\mu v}, \mathbf{I}_{\mu}, \partial_{\tau}, \tau \partial_{\tau}
$$

Consequently, for the $(3+1)$-dimensional decomposable space

$$
d s^{2}=-\varepsilon d \tau^{2}+\frac{1}{\left(1+\frac{1}{4} K x^{i} x_{i}\right)^{2}}\left(d x^{2}+d y^{2}+d z^{2}\right)
$$

it follows that the vector fields $\mathbf{r}_{\mu v}, \mathbf{I}_{\mu}$ are KVs, $\partial_{\tau}$ is a gradient $K V$, while $\tau \partial_{\tau}$ is an AC.

For a second application, let us assume the four-dimensional Gödel spacetime in Cartesian coordinates

$$
d \tau^{2}=-d t^{2}-2 e^{a x} d t d y+d x^{2}-\frac{1}{2} e^{2 a x} d y^{2}+d z^{2} .
$$

The geodesic equations are

$$
\begin{aligned}
t^{\prime \prime}+2 a t^{\prime} x^{\prime}+a e^{a x} x^{\prime} y^{\prime} & =0, \\
x^{\prime \prime}+a e^{a x} t^{\prime} y^{\prime}+\frac{1}{2} a e^{2 a x} y^{\prime 2} & =0, \\
y^{\prime \prime}-2 a e^{-a x} t^{\prime} x^{\prime} & =0, \\
z^{\prime \prime} & =0 .
\end{aligned}
$$

The Gödel metric admits the following elements for the special projective algebra

$$
\begin{gathered}
Y^{1}=\partial_{z}, Y^{3}=\partial_{x}-a y \partial_{y}, Y^{4}=\partial_{t}, Y^{5}=\partial_{y}, Y^{6}=z \partial_{z} \\
Y^{2}=\left(-\frac{2}{a} e^{-a x}\right) \partial_{t}+y \partial_{x}+\left(\frac{2 e^{-2 a r}-a^{2} y^{2}}{2 a}\right) \partial_{y}
\end{gathered}
$$

where $Y^{1}$ is a gradient $\mathrm{KV}\left(S_{1}=z\right), Y^{2-5}$ are non gradient $\mathrm{KVs}$ and $Y^{6}$ is a proper AC. Consequently, the Lie point symmetries are derived to be

$$
\begin{gathered}
X_{1}=\partial_{\tau}, X_{2}=\tau \partial_{\tau}, X_{3}=z \partial_{\tau}, X_{4}=Y^{4} \\
X_{5}=Y^{2}, X_{6}=Y^{3}, X_{7}=Y^{5} \\
X_{8}=Y^{1}, X_{9}=\tau Y^{1}, \quad X_{10}=Y^{6}
\end{gathered}
$$

Thus, for the five-dimensional line element

$$
d \tau^{2}=\varepsilon d \tau^{2}-d t^{2}-2 e^{a x} d t d y+d x^{2}-\frac{1}{2} e^{2 a x} d y^{2}+d z^{2}
$$

vector field $X_{1}$ is a gradient $\mathrm{KV}, X_{2}, X_{3}$ and $X_{9}$ are proper ACs, $X_{4-10}$ have the same properties as that for the four-dimensional manifold. We observe that the field $X_{3}-\varepsilon X_{9}$ is a rotation on the directions $\tau-z$, which is a Killing symmetry for the five-dimensional space. 
As a final example consider the three-dimensional line element

$$
d x_{(3)}^{2}=-d t^{2}+(a t+b)^{2}\left(d y^{2}+d z^{2}\right) .
$$

which admits the three nongradient KVs

$$
K^{1}=\partial_{y}, K^{2}=\partial_{z}, K^{3}=z \partial_{y}-y \partial_{z} .
$$

and the gradient HKV

$$
H^{i}=\left(t+\frac{b}{a}\right) \partial_{t}, \psi=1
$$

Thus, the Lie point symmetries for the geodesic equations for the space are the vector fields

$$
\partial_{x}, x \partial_{x}, K^{1}, K^{2}, K^{3}, H^{i}, x^{2} \partial_{x}+x H^{i} .
$$

Hence, for the four-dimensional space

$$
d s^{2}=-d t^{2}+d x^{2}+(a t+b)^{2}\left(d y^{2}+d z^{2}\right)
$$

it follows that $K^{1}, K^{2}, K^{3}$ are nongradient $K V s, \partial_{x}$ is a gradient $\mathrm{KV}, x \partial_{x}+H^{, i}$ is the gradient $\mathrm{HKV}$; $x \partial_{x}, H^{i}$ are ACs. Finally, $x^{2} \partial_{x}+x H^{i}$ is a proper special PC for the decomposable spacetime.

\section{Conclusions}

The theory of symmetries for differential equations is important for the study of the integrability properties and the determination of conservation laws. The latter can be used to understand the trajectories of geometrical objects such as the orbits of astrophysical objects $[62,63]$. Furthermore, the procedure for the derivation of the Lie symmetries is straightforward, but is usually a high dimension system and symbolic computation software is usually applied [64]. In this study, we reviewed previous published results for the geometric description and construction of the Lie point symmetries. This approach connects the two different studies between the geometric properties of Riemannian manifolds and of the symmetries for differential equations. We show that important results of differential geometry can be derived easily by using the Lie symmetry analysis.

In this spirit, we focused on the geometric interpretation of the Lie point symmetries for the geodesic equations. We proved that the Lie point symmetries are the elements of the projective algebra for an extended decomposable space, while the projective algebra is directed related with the special projective algebra of the nondecomposable space. The existence criteria for the nature of specific geometric collineations, such as the rotations, the proper ACs or the proper special PC discussed in details.

This study contributes to the subject for the geometrization of the symmetries of differential equations. Such a geometric approach has also been applied in the case of partial differential equations where different relations between the Lie point symmetries and the collineations of the background space were found [65-67]. This geometric approach can be seen as a connection bridge between the differential geometry and the theories of differential equations and applied mathematics. In this study, we show an alternative way for the study of existence theorems; in the future, we plan to investigate further applications for this geometric point of view.

Funding: This work is based on the research supported in part by the National Research Foundation of South Africa (Grant Numbers 131604).

Institutional Review Board Statement: Not applicable.

Informed Consent Statement: Not applicable.

Data Availability Statement: Not applicable. 
Conflicts of Interest: The author declares no conflict of interest.

\section{References}

1. Lie, S. Theorie der Transformationsgruppen: Volume I; Nabu Press: Chelsea, NY, USA, 1970.

2. Lie, S. Theorie der Transformationsgruppen: Volume II; Nabu Press: Chelsea, NY, USA, 1970.

3. Lie, S. Theorie der Transformationsgruppen: Volume III; Nabu Press: Chelsea, NY, USA, 1970.

4. Lewis, H.R.; Leach, P.G.L. Exact invariants for a class of time-dependent nonlinear Hamiltonian systems. J. Math. Phys. 1982, $23,165$. [CrossRef]

5. Mahomed, F.M. Symmetry group classification of ordinary differential equations: Survey of some results. Math. Meth. Appl. Sci. 2007, 30, 1995. [CrossRef]

6. Cherniha, R.; Davydovych, V.; King, J.R. Lie Symmetries of Nonlinear Parabolic-Elliptic Systems and Their Application to a Tumour Growth Model. Symmetry 2018, 10, 171. [CrossRef]

7. Craddock, M. Symmetry Groups of Linear Partial Differential Equations and Representation Theory: The Laplace and Axially Symmetric Wave Equations. J. Differ. Equ. 2000, 166, 107. [CrossRef]

8. Webb, G.M. Lie symmetries of a coupled nonlinear Burgers-heat equation system. J. Phys. A Math. Gen. 1990, 23, 3885. [CrossRef]

9. Chesnokov, A. Symmetries and exact solutions of the rotating shallow-water equations. Eur. J. Appl. Math. 2009, 20, 461. [CrossRef]

10. Paliathanasis, A. One-Dimensional Optimal System for 2D Rotating Ideal Gas. Symmety 2019, 11, 1115. [CrossRef]

11. Paliathanasis, A. Lie symmetries and singularity analysis for generalized shallow-water equations. Int. J. Nonlinear Sci. Numer. Simul. 2020, 20, 739. [CrossRef]

12. Tsamparlis, M.; Paliathanasis, A. Symmetries of Differential Equations in Cosmology. Symmetry 2018, 10, 233. [CrossRef]

13. Bihlo, A.; Poltavets, N.; Popovych, R.O. Lie symmetries of two-dimensional shallow water equations with variable bottom topography. Chaos Interdiscip. J. Nonlinear Sci. 2020, 30, 073132. [CrossRef]

14. Papamikos, G.; Pryer, T. A Lie symmetry analysis and explicit solutions of the two-dimensional $\infty$-Polylaplacian. Stud. Appl. Math. 2019, 142, 48. [CrossRef]

15. Andriopoulos, K.; Dimas, S.; Leach, P.G.L.; Tsoubelis, D. On the systematic approach to the classification of differential equations by group theoretical methods. J. Comput. Appl. Math. 2009, 230, 224. [CrossRef]

16. Ahmad, A.; Bokhari, A.H.; Kara, A.H.; Zaman, F.D. Symmetry reductions for 2-dimensional non-linear wave equation. Nuovo Cimento B 2006, 121, 571.

17. Bokhari, A.H.; Johnpillai, A.G.; Mahomed, F.M.; Zaman, F.D. Approximate conservation laws of nonlinear perturbed heat and wave equations. Nonlinear Anal. Real World Appl. 2012, 13, 2823. [CrossRef]

18. Nucci, M.C. Using Lie symmetries in Epidemiology. Electron. J. Differ. Equ. 2015, 12, 87.

19. Gazizov, R.K.; Ibragimov, N.H. Lie Symmetry Analysis of Differential Equations in Finance. Nonlinear Dyn. 1998, $17,387$. [CrossRef]

20. O'Hara, J.G.; Sophocleous, C.; Leach, P.G.L. Application of Lie point symmetries to the resolution of certain problems in financial mathematics with a terminal condition. J. Eng. Math. 2013, 82, 67. [CrossRef]

21. Bilho, A.; Popovych, R.O. Lie symmetry analysis and exact solutions of the quasigeostrophic two-layer problem. J. Math. Phys. 2011, 52, 033103.

22. Mahomed, F.M.; Qadir, A. Linearization criteria for a system of second-order quadratically semi-linear ordinary differential equations. Nonlinear Dyn. 2007, 48, 417. [CrossRef]

23. Bluman, G.W. Potential Symmetries and Linearization. In Applications of Analytic and Geometric Methods to Nonlinear Differential Equations; Clarkson, P.A., Ed.; NATO ASI Series (Series C: Mathematical and Physical Sciences); Springer: Dordrecht, The Netherlands, 1993; Volume 413.

24. Ali, S.; Safader, M.; Qadir, A. Linearization from Complex Lie Point Transformations. J. Appl. Math. 2014, 2014, 793247. [CrossRef]

25. Al-Dweik, A.Y.; Mustafa, M.T.; Mara'Beh, R.A.; Mahomed, F.M. An alternative proof of Lie's linearization theorem using a new $\lambda$ -symmetry criterion. Commun. Nonlinear Sci. Numer. 2015, 26, 45. [CrossRef]

26. LOvsiannikov, V. Group Analysis of Differential Equations; Academic Press: New York, NY, USA, 1982.

27. Maartens, R.; Mason, D.P.; Tsamparlis, M. Kinematic and dynamic properties of conformal Killing vectors in anisotropic fluids. J. Math. Phys. 1986, 27, 2987. [CrossRef]

28. Coley, A.A.; Tupper, B.O.J. Affine conformal vectors in space-time. J. Math. Phys. 1992, 33, 1754. [CrossRef]

29. Coley, A.A.; Tupper, B.O.J. Spherically symmetric anisotropic fluid ICKV spacetimes. Class. Quantum Gravity 1994, $11,2553$. [CrossRef]

30. Maartens, R.; Maharaj, S.D.; Tupper, B.O.J. General solution and classification of conformal motions in static spherical spacetimes. Class. Quantum Gravity 1995, 12, 2577. [CrossRef]

31. Faraoni, V. A Symmetry of the Einstein-Friedmann Equations for Spatially Flat, Perfect Fluid, Universes. Symmetry 2020, $12,147$. [CrossRef]

32. Hall, G.S.; Lonie, D.P. Projective collineations in spacetimes. Class. Quantum Gravity 1995, 12, 1007. [CrossRef]

33. Frances, C. Local dynamics of conformal vector fields. Geom. Dedicata 2012, 158, 35. [CrossRef]

34. Joharinad, P.; Bidabad, B. Conformal vector fields on Finsler spaces. Differ. Geom. Appl. 2013, 31, 33. [CrossRef] 
35. Geroch, R. A Method for Generating New Solutions of Einstein's Equation. I. J. Math. Phys. 1971, 12, 918. [CrossRef]

36. Geroch, R. A Method for Generating New Solutions of Einstein's Equation. II. J. Math. Phys. 1972, 13, 394. [CrossRef]

37. Canfora, F.; Dimakis, N.; Giacomini, A.; Paliathanasis, A. Bianchi IX cosmologies in the Einstein-Skyrme system in a sector with nontrivial topological charge. Phys. Rev. D 2019, 99, 044035. [CrossRef]

38. Ayon-Beato, E.; Canfora, F.; Zanelli, J. Analytic self-gravitating Skyrmions, cosmological bounces and AdS wormholes. Phys. Lett. B 2016, 752, 201. [CrossRef]

39. Canfora, F.; Paliathanasis, A.; Zanelli, J. Cosmological Einstein-Skyrme solutions with nonvanishing topological charge. Phys. Rev. D 2017, 95, 065032. [CrossRef]

40. Hall, G.S.; Low, D.J.; Rulham, J.R. Affine collineations in general relativity and their fixed point structure. J. Math. Phys. 1994, 35, 5930. [CrossRef]

41. Katzin, G.H.; Levine, J. Dynamical symmetries and constants of the motion for classical particle systems. J. Math. Phys. 1974, 15, 1460. [CrossRef]

42. Katzin, G.H.; Levine, J. A gauge invariant formulation of time-dependent dynamical symmetry mappings and associated constants of motion for Lagrangian particle mechanics. I. J. Math. Phys. 1976, 17, 1345. [CrossRef]

43. Katzin, G.H.; Levine, J. Geodesic first integrals with explicit path-parameter dependence in Riemannian space-times. J. Math. Phys. 1981, 22, 1878. [CrossRef]

44. Aminova, A.V. Projective transformations and symmetries of differential equations. Sb. Math. 1995, 186, 1711. [CrossRef]

45. Feroze, T.; Mahomed, F.M.; Qadir, A. The Connection Between Isometries and Symmetries of Geodesic Equations of the Underlying Spaces. Nonlinear Dyn. 2006, 45, 65. [CrossRef]

46. Bokhari, A.H.; Kara, A.H.; Kashif, A.R.; Zaman, F.D. Noether Symmetries Versus Killing Vectors and Isometries of Spacetimes. Int. J. Theor. Phys. 2006, 45, 1029. [CrossRef]

47. Bokhari, A.H.; Johnpillai, A.G.; Kara, A.H.; Mahomed, F.M.; Zaman, F.D. Classification of Static Spherically Symmetric Spacetimes by Noether Symmetries. Int. J. Theor. Phys. 2013, 52, 3534. [CrossRef]

48. Bokhari, A.H.; Kara, A.H. Noether versus Killing symmetry of conformally flat Friedmann metric. Gen. Relativ. Gravit. 2007, 39 , 2053. [CrossRef]

49. Battle, C.; Gomis, J.; Ray, S.; Zanelli, J. Lie Symmetries of Non-Relativistic and Relativistic Motions. Phys. Rev. D 2019, 98, 064015. [CrossRef]

50. Tsamparlis, M.; Paliathanasis, A. Lie symmetries of geodesic equations and projective collineations. Nonlinear Dyn. 2010, 62, 203. [CrossRef]

51. Tsamparlis, M.; Paliathanasis, A. Lie and Noether symmetries of geodesic equations and collineations. Gen. Relativ. Gravit. 2010, $42,2957$. [CrossRef]

52. Tsamparlis, M.; Paliathanasis, A. The geometric nature of Lie and Noether symmetries. Gen. Relativ. Gravit. $2011,43,1861$. [CrossRef]

53. Tsamparlis, M.; Mitsopoulos, A. First integrals of holonomic systems without Noether symmetries. J. Math. Phys. 2020, 61, 122701. [CrossRef]

54. Tsamparlis, M.; Mitsopoulos, A. Quadratic first integrals of autonomous conservative dynamical systems. J. Math. Phys. 2020, 61, 072703. [CrossRef]

55. Stephani, H. Differential Equations: Their Solutions Using Symmetry; Cambridge University Press: New York, NY, USA, 1989.

56. Yano, K. Lie Derivatives and Its Applications; North-Holand Publishing Co.: Amsterdam, The Netherlands, 1995.

57. Barnes, A. Projective collineations in Einstein spaces. Class. Quantum Gravity 1993, 10, 1139. [CrossRef]

58. Katzine, G.H.; Levine, J.; Davis, W.R. Curvature Collineations: A Fundamental Symmetry Property of the Space-Times of General Relativity Defined by the Vanishing Lie Derivative of the Riemann Curvature Tensor. J. Math. Phys. 1969, 10, 617. [CrossRef]

59. Cariñena, J.F.; de Lucas, J.; Rañada, M.F. Jacobi multipliers, non-local symmetries and nonlinear oscillators. J. Math. Phys. 2015, 56, 063505. [CrossRef]

60. Hojman, S.A. A new conservation law constructed without using either Lagrangians or Hamiltonians. J. Phys. A Gen. Phys. 1992, 25, L291. [CrossRef]

61. Zhang, H.-B.; Chen, L.-Q. The Unified Form of Hojman's Conservation Law and Lutzky's Conservation Law. J. Phys. Soc. Jpn. 2005, 74, 905. [CrossRef]

62. Al-Ali, U.; Bokhari, A.H.; Kara, A.H.; Shabbir, G. Classification of Variational Conservation Laws of General Plane Symmetric Spacetimes. Commun. Theor. Phys. 2017, 68, 335. [CrossRef]

63. Akhtar, S.; Hussain, T.; Bokhari, A.H. Positive Energy Condition and Conservation Laws in Kantowski-Sachs Spacetime via Noether Symmetries. Symmetry 2018, 10, 712. [CrossRef]

64. Hereman, W. Review of symbolic software for lie symmetry analysis. Math. Comput. Model. 1997, 25, 115. [CrossRef]

65. Paliathanasis, A.; Tsamparlis, M. The geometric origin of Lie point symmetries of the Schrödinger and the Klein-Gordon equations. Int. J. Geom. Methods Mod. Phys. 2014, 11, 1450037. [CrossRef]

66. Aslam, A.; Qadir, A. Noether Symmetries of the Area-Minimizing Lagrangian. J. Appl. Math. 2012, 2012, 532690. [CrossRef]

67. Bozhkov, Y.; Freire, I.L. Special conformal groups of a Riemannian manifold and Lie point symmetries of the nonlinear Poisson equation. J. Differ. Equ. 2010, 249, 872. [CrossRef] 